\title{
A VGA tachistoscope
}

\author{
GARY P. FINLEY \\ University of Alberta, Edmonton, Alberta, Canada
}

\begin{abstract}
A set of tachistoscope-emulation functions for IBM-PC-compatible computers equipped with the VGA display controller is described. $\mathrm{C}$ or Pascal programs including these functions can easily produce multifield tachistoscopic presentations of colored text.
\end{abstract}

Microcomputers can easily produce dynamic displays of text or pictures to emulate the functions of a traditional electric tachistoscope. Simple text-presentation tasks can be carried out with the character display routines built into computer languages such as BASIC, Pascal, and C. However, such generic text-output software is not designed for fast display manipulations, and it does not include all of the functions that are required for high-quality tachistoscope emulation.

\section{Tachistoscope Functions}

In a previous paper (Finley, 1989), I described a package of software in the $\mathrm{C}$ and Pascal languages for tachistoscope emulation on IBM-PC-compatible computers equipped with the monochrome/graphics display controller made by Hercules Computer Technology (2550 Ninth St., Berkeley, CA 94710). This software package includes functions for fast-paced presentations of text stimuli, as well as functions for screen blanking, for cursor control, and for synchronizing display changes with the monitor's video scan. I designed the software to achieve fast screen switching by storing virtual screens of text in unused areas of the display controller's video memory. Any of these prestored screens can be selected for display by changing the value stored in the display controller register that selects the portion of video memory that appears on the monitor. This register manipulation is much faster than changing the text stored in a single area of the video controller's memory. The time required to change from one virtual screen to another is independent of the number of characters stored. Screens containing a full 2,000 characters ( 25 rows of 80 column text) can be switched in the same time (typically $50 \mu \mathrm{sec})$ as can screens containing a single character.

\section{The VGA Display Controller}

In 1987, IBM (900 King Dr., Rye Brook, NY 10573) introduced its PS/2 family of computers, including a highresolution display system based on the VGA (Video Graphics Array) controller. Inexpensive VGA-compatible display controllers are now available for all brands of IBMcompatible computers. VGA displays have better resolu-

Correspondence may be addressed to Gary P. Finley, Psychology Department, University of Alberta, Edmonton, Alberta T6G 2E9, Canada. tion than those produced by earlier IBM video controllers like the Color Graphics Adapter (CGA) and Enhanced Graphics Adapter (EGA), and they can produce a greater number of colors in some display modes. VGA controllers can drive inexpensive monochrome monitors, offering a good alternative to Hercules displays for cost-conscious users. Programs written for a VGA controller will work with both monochrome and color VGA monitors without modification. This is an important advance over the CGA, EGA, and Hercules controllers, which require specialized (and mutually incompatible) display-driver software. When displayed on a monochrome screen, the colors of a VGA image are rendered as levels of monochromatic brightness.

\section{VGA Tachistoscope Functions}

I have revised the Hercules tachistoscope-emulation function package for use with VGA display controllers. The new software includes several enhancements made possible by the VGA's advanced display-control hardware. These new features include the following:

1. The VGA tachistoscope has eight fields. The Hercules controller limited the number of virtual screens to four, but the VGA display controller allows eight virtual screens of 2,000 characters to be simultaneously stored in display memory.

2. Both text and background colors are under program control. The new functions provide control over the color of the displayed characters and the screen background. Separate color assignments can be made in each virtual screen. Sixteen colors can be displayed at one time, selected from a range of 256,000 choices. A public-domain program is available (Gerrold, 1990) for convenient color selection through adjustment of the VGA palette registers. If a monochrome VGA monitor is used, these color selections control the brightness of the displayed text and the contrast between the text and the background.

3. The text font can be customized by the user. The VGA's built-in font can be replaced by a file of characterfont data supplied by the host computer. This is similar to the process of downloading a font to a laser printer. Custom-designed text fonts open up many display possibilities, such as non-English or nonsense characters, inverted or mirror-image characters, or nonalphabetic geometric shapes. Another interesting option is the display 
of large characters formed by a grid of adjacent character cells, each of which displays a segment of an enlarged character. A public-domain program (Mefford, 1988) can be used to create and modify VGA font files. This program produces executable files that load a customized font into the VGA controller, where it becomes available to any program (including the tachistoscope functions) that displays text on the screen.

VGA controllers are well suited to tachistoscope emulation. All of the necessary functions (virtual screen storage, display blanking, cursor control, and video synchronization) are included in the VGA's Basic Input/Output Software (BIOS), which is stored in a read-only memory on the VGA circuit board. In the Hercules, CGA, and EGA controllers, a complete set of tachistoscope functions cannot be created without programming the display controller's registers directly. Programs that use this approach may not work equally well on all display controllers, because of internal hardware differences. This problem should not arise with the VGA tachistoscope functions, since they access the VGA controller through its BIOS software.

\section{Availability}

A printed listing of the VGA tachistoscope functions and a demonstration program will be sent on request. To obtain a copy of the files on disk (5.25 or 3.5 in.), send a blank, formatted disk to the author. Please specify the $C$ or Pascal version of the package. In addition, a description of how the functions could be recoded into subroutines in BASIC will be included. The author may be contacted on e-mail at USERGFSO@MTS.UCS.UALBERTA.CA.

\section{REFERENCES}

FinLEy, G. P. (1989). Tachistoscopic software for the Hercules display controller. Behavior Research Methods, Instruments, \& Computers, 21, 387-390.

Gerrold, D. (1990). Free your VGA from CGA captivity with Prism.exe. PC Magazine, 9 (21), 463-480.

Mefrord, M. J. (1988). Making your own screen fonts. PC Magazine, 7 (15), 305-324.

(Manuscript received February 15, 1991; revision accepted for publication June 3,1991 .)

\section{Call for Assistance in The Compilation of a History of the Psychonomic Society}

The Governing Board of the Psychonomic Society is pleased to announce that Robert C. Bolles has agreed to serve as the first Historian of the Society.

All members who might have information relevant to this undertaking are invited to send it directly to Dr. Bolles. Founding members and those who attended the early meetings are especially encouraged to record their reminiscences. While Dr. Bolles hopes to collect as much information as possible relevant to the history of the Society, he will concentrate first on the early history.

Dr. Bolles's address is Department of Psychology, University of Washington, Seattle, Washington 98195 (phone: 206-543-2631). 